\title{
The effect of chronic tobacco smoking on arterial stiffness
}

\author{
Nirandeep Rehill, Charles R. Beck, Karen Rowland Yeo \& Wilfred W. Yeo \\ Academic Unit of Clinical Pharmacology, Division of Clinical Sciences (South), University of Sheffield, Royal Hallamshire Hospital, Sheffield, \\ $U K$
}

\begin{abstract}
Correspondence
Dr W. W. Yeo, Academic Unit of Clinical Pharmacology, Division of Clinical Sciences (South), University of Sheffield, Royal Hallamshire Hospital, Sheffield S10 2JF, UK.

Tel.: + 44 (0)1142713176

Fax: + $44(0) 1142268986$

E-mail:w.w.yeo@sheffield.ac.uk
\end{abstract}

Source of funding: this study was funded internally by Dr W. Yeo, University of Sheffield.

\section{Keywords \\ arteries, endothelium, nitric oxide, smoking}

\section{Received}

15 September 2005

Accepted

16 December 2005

Published OnlineEarly

27 February 2006

\begin{abstract}
Aims
Cardiovascular disease caused by smoking is related to the pathophysiological burden placed on the vascular endothelium. We studied the effect of chronic cigarette smoking on arterial wave reflection (study 1) and smoking cessation on pulse wave analysis (study 2).
\end{abstract}

\begin{abstract}
Methods
Fifty smokers and 50 age- and sex-matched nonsmokers participated in study 1. Study 2 recruited 20 volunteers from the stop smoking clinic at the Royal Hallamshire Hospital, Sheffield, UK. Systemic augmentation index (Alx) and carotid-femoral pulse wave velocity (PWV) were measured using the SphygmoCor system. Brachial blood pressure (BP) (Omron 705-CP-E), Alx and PWV were recorded at a single visit in study 1 . Study 2 measured these variables on 'quit day' and 4 weeks later.
\end{abstract}

\section{Results}

In study 1, Alx was significantly higher in smokers than in nonsmokers (median 17.25 vs. $11.75 \%, P=0.004$ ). Multiple regression analysis showed a significant correlation between Alx and age, diastolic BP, smoking status $(P<0.001)$, blood glucose $(P=0.045)$ and weight $(P=0.049)$. In study 2 , Alx significantly reduced after 4 weeks of abstinence in successful quitters $(n=10)$ compared with relapsed smokers $(n=4)$ (median 5.0 vs. $-9.5 ; P=0.013$ ). PWV did not reach significance in either study.

\section{Conclusions}

Chronic tobacco smoking is associated with endothelial dysfunction and increased Alx in subjects of a wide age range free from additional cardiovascular risk factors, which is partially reversible after 4 weeks of smoking cessation.

\section{Introduction}

Smoking is one of the leading modifiable risk factors for cardiovascular disease [1]. Much of the increased cardiovascular risk caused by smoking may be due to adverse effects on the vascular endothelium, induction of coronary vasoconstriction and changes in basal nitric oxide (NO) or endothelial nitric oxide synthase (eNOS) protein production [1-3]. Mahmud and Feely have demonstrated that smoking a single cigarette produces an acute increase in arterial stiffness in chronic smokers and nonsmokers [4]. Vlachopoulos et al. have shown similar findings where a significant undesirable combined effect of smoking and caffeine was found on arterial stiffness [5]. Increased carotid arterial stiffness and systemic augmentation index (AIx) have also been previously reported following 
exposure to environmental tobacco smoke in nonsmokers $[6,7]$.

The risk of myocardial infarction (a major endpoint of cardiovascular disease) is known to return to normal levels within a few years of quitting smoking, suggesting that the cardiovascular damage caused by smoking may be reversible [8]. It may therefore be hypothesized that reduced basal NO secretion associated with smoking is also reversible and that endothelial function returns to normal after quitting smoking. Measurement of endothelial dysfunction may be a potential target for cardiovascular risk factor modification [9]. Arterial stiffness is emerging as an important cardiovascular risk factor predominately due to new non-invasive technologies which enable measurements to be taken in largescale clinical trials. The shape of the arterial pressure waveform provides a measure of systemic arterial stiffness and can be assessed using the technique of pulse wave analysis (PWA) [10]. It has been demonstrated as a reproducible method for determining AIx and pulse wave velocity (PWV) [10,11]. Arterial stiffness can be used as a surrogate measure of endothelial function since it is partially dependent on vascular smooth muscle tone $[12,13]$. PWV measures large artery stiffness; carotid-femoral PWV is considered to be the most clinically relevant because the aorta and its first branches are responsible for regulating blood pressure in the periphery and maintaining diastolic coronary artery flow. PWA has previously been used to demonstrate increased arterial wave reflection in a cohort of healthy young smokers compared with nonsmokers [4].

We have performed two studies to investigate the effect of smoking on arterial stiffness. Study 1 investigated the effect of chronic cigarette smoking in healthy volunteers aged 18-60 years on systemic AIx and PWV in 50 smokers vs. 50 age- and sex-matched nonsmoking controls. Study 2 collected preliminary data describing the effect of smoking cessation on arterial stiffness and endothelial function using PWA at baseline and 4 weeks post quit day.

\section{Methods}

\section{Subject population}

Study 1 One hundred volunteers aged between 18 and 60 years (mean \pm SD $37.9 \pm 11.4$ ) with a body mass index (BMI) of 19.2-39.2 (25.8 \pm 3.9$)$ participated in the study. Fifty were smokers who had smoked 10 cigarettes or more $(16.2 \pm 5.1)$ per day for at least 1 year prior to recruitment. The remaining 50 volunteers were age- (within 5 years) and sex-matched nonsmokers who had not smoked at all over the past year. Thirteen had been smokers, with 10 stopping between 5 and 27 years and three between 1.5 and 5 years before recruitment. All participants were screened by medical history and blood tests [serum total cholesterol, high-density lipoprotein (HDL)-cholesterol, glucose, creatinine, Creactive protein $(\mathrm{CRP})]$ to exclude those with preexisting disease that may have confounded the PWA results [10]. Serum nicotine and cotinine levels were determined by mass spectrometry based on the method described by Stolker et al. [14]. Smokers had their smoking status confirmed by expired carbon monoxide (CO) testing (Bedfont Scientific Ltd, Rochester, UK). A subset of volunteers ( $n=26$ matched pairs) provided blood samples for high-sensitivity CRP analysis (hsCRP; Biocheck, Inc., Burlingame, CA, USA). The study protocol had local ethics committee approval and all subjects provided written informed consent.

Study 2 Twenty patients who were enrolled into the stop smoking clinic at the Royal Hallamshire Hospital, Sheffield, UK took part in the study. Subjects were aged $57.2 \pm 10.4$ years and had a BMI of 27.9 \pm 5.7 . Eight $(40 \%)$ subjects had established cardiovascular disease, $12(60 \%)$ had respiratory disease and were taking $6.1 \pm 5.6$ concomitantly prescribed drugs. At baseline, subjects smoked 22.2 \pm 9.4 cigarettes per day for $37.1 \pm 17.3$ years, had a mean heart rate of $66 \pm 11$ beats per minute and brachial blood pressure of 127/76 \pm 15 / $9 \mathrm{mmHg}$. All subjects receiving nitrate medication were excluded, but other cardiovascular drugs were allowed as patients were acting as their own control (drug treatment was kept constant throughout the study). Successful quitters were defined as patients who were not smoking by 4 weeks after quit date and had used less than 10 cigarettes during this time. The study protocol had local ethics committee approval and all subjects provided written informed consent.

\section{Experimental protocol}

Study 1 To avoid measuring the acute effects of smoking and dietary nitrates, subjects fasted for $12 \mathrm{~h}$ from food, smoking, alcohol and caffeine-containing beverages. Subjects were studied supine in a quiet room after 5 min rest. PWA was performed at the radial pulse to determine AIx and the carotid and femoral pulses to determine PWV. All measurements were repeated, with AIx measurements standardized for heart rate at 75 beats per minute [10].

Study 2 Patients were asked to attend study visits on quit day and 4 weeks later. A detailed dietary history of 
the previous $48 \mathrm{~h}$ was obtained at baseline in order to give an indication of dietary nitrate consumption. All volunteers were asked to eat an equivalent nitrate diet during the $48 \mathrm{~h}$ before their next study visit, and to follow a similar procedure for caffeine and alcohol consumption. Smoking status was assessed and documented at each visit.

Blood pressure measurements Brachial blood pressure (BP) was recorded supine in a quiet room at room temperature after 5 min rest using an automated digital oscillometric BP monitor (Omron 705-CP-E; Omron Corp., Tokyo, Japan). Three readings separated by 1min intervals were taken and the mean of the last two readings were recorded. The estimated arterial BP was determined following analysis of the pressure waveform by the SphygmoCor software version 6.31 (AtCor Medical, Sydney, Australia).

Analysis of the arterial pressure waveform AIx and PWV were determined by applanation tonometry following BP measurements. A high-fidelity micro pressure probe (SPC-301; Millar Instruments, Houston, TX, USA) was used to record systemic arterial stiffness at the radial artery and aortic pulse wave velocity by analysing the arterial pressure wave at the carotid and femoral arteries. The SphygmoCor software automatically captures the pressure waveforms and has a built-in quality control [10], and has been used to record smokingrelated changes in arterial stiffness $[4,5,7]$.

\section{Statistical analyses}

In Study 1, AIx and PWV were studied between matched pairs using the Wilcoxon signed-rank nonparametric test. Multiple regression analysis was used to identify factors associated with changes in AIx and PWV. We were unable to obtain AIx readings from $n=2$ subjects, PWV readings from $n=12$ subjects and were missing data for one or more of the regression model predictors from $n=10$ subjects, which is consistent with other PWA studies [15]. Two matched pairs were excluded from the Wilcoxon AIx analysis $(n=96)$ and 10 matched pairs were excluded from the PWV analysis $(n=80)$. A sample size of $n=88$ was employed in the AIx model and $n=78$ subjects were included in the PWV model. High-sensitivity CRP levels were compared between a subset of smokers and nonsmokers using the Wilcoxon signed-rank test $(n=52)$. In study 2 , subjects were divided into successful quitters and relapsed smokers. Changes in AIx and PWV between baseline and 4 weeks post quit day were analysed using the Mann-Whitney nonparametric test. Six patients did not attend their 4-week study visits and were assumed to have gone back to regular tobacco smoking. Five successful quitters and two relapsed smokers were excluded from the PWV analyses $(n=7)$ due to difficulties palpating the arterial study sites. Minitab ${ }^{\mathrm{TM}}$ software version 14.12.0 (Minitab Inc., State College, PA, USA) was used to carry out all statistical tests. $\alpha=0.05$ was used in all cases.

\section{Results}

Study 1

Baseline characteristics of the volunteers are shown in Table 1. There was no significant difference in age, gender, height, weight, BMI, creatinine, glucose, hsCRP or total cholesterol between the matched pairs, although the smokers did have a significantly lower HDLcholesterol, higher total : HDL-cholesterol ratio and a higher alcohol intake than the nonsmokers. AIx in smokers was significantly higher than that in nonsmokers [median 17.25 vs. $11.75 \%$, median difference 5.5, 95\% confidence interval (CI) 2.0, 8.75; $P=0.004$ ]. Figure 1 demonstrates the increasing proportion of smokers with higher AIx readings compared with nonsmokers. However, PWV was not significantly different between the two groups (median nonsmokers $5.6 \mathrm{vs}$. smokers $5.9 \mathrm{~m} \mathrm{~s}^{-1}$, median difference $0.225,95 \%$ CI -0.55 , $0.075 ; P=0.15)$.

Multiple regression analysis demonstrated a significant positive association between AIx and age, diastolic BP and smoking status, and a negative association with glucose and weight (Table 2). The model explained $78.3 \%$ of the variability in AIx observed in the study $(P<0.001)$. Multiple regression analysis for PWV investigating the same predictors as for AIx and including height [16] showed that age was the only significant predictor $(P<0.001)$.

Matched-pair analysis in the 52 subjects with serum hsCRP levels did not show a significant difference between nonsmokers and smokers (median nonsmokers 1.2 vs. smokers $1.5 \mathrm{mg} \mathrm{l}^{-1}$, median difference $0.10,95 \%$ CI $-0.79,0.42, P=0.48$ ). Univariate regression analysis indicated that hsCRP levels independently predicted aortic PWV $(P=0.028)$ and approached significance for AIx $(P=0.059)$.

\section{Study 2}

At 4 weeks post quit day, 10 patients had successfully reduced their smoking to $<10$ cigarettes over the 4-week period ( 0 cigarettes, $n=6 ; 1-3$ cigarettes, $n=2 ; 4-6$ cigarettes, $n=2)$. Four patients had smoked 10 or more cigarettes during this time (10-20 cigarettes, $n=2 ; 20+$ cigarettes, $n=2$ ). Six patients did not attend the 4-week follow-up study visit and were withdrawn from the study. 
N Rehill et al.

Table 1

Study 1: subjects' baseline characteristics

\begin{tabular}{|c|c|c|c|}
\hline & $\begin{array}{l}\text { Smokers } \\
(n=50)\end{array}$ & $\begin{array}{l}\text { Nonsmokers } \\
(n=50)\end{array}$ & $P$-value \\
\hline Age (years) & $37.8 \pm 11.8$ & $38.1 \pm 11.1$ & \\
\hline Height (cm) & $1.7 \pm 0.1$ & $1.7 \pm 0.1$ & \\
\hline Weight (kg) & $74.6 \pm 15.8$ & $71.7 \pm 12.6$ & \\
\hline Creatinine $\left(\mathrm{mmol} \mathrm{l}^{-1}\right)$ & $74.1 \pm 13.4$ & $77.9 \pm 15.8$ & \\
\hline Glucose $\left(\mathrm{mmol} \mathrm{I}{ }^{-1}\right)$ & $5.3 \pm 1.1$ & $5.0 \pm 0.8$ & \\
\hline \multicolumn{4}{|l|}{ Cholesterol } \\
\hline Total cholesterol (mmol l-1) & $5.1 \pm 1.1$ & $4.8 \pm 1.1$ & \\
\hline HDL-cholesterol $\left(\mathrm{mmol} \mathrm{I}^{-1}\right)$ & $1.3 \pm 0.4$ & $1.4 \pm 0.4$ & 0.012 \\
\hline Total:HDL ratio & $4.3 \pm 1.6$ & $3.5 \pm 1.0$ & 0.001 \\
\hline Pack years smoked & $16.4 \pm 12.7$ & $1.3 \pm 3.8$ & $<0.001$ \\
\hline \multicolumn{4}{|l|}{ Haemodynamics } \\
\hline Heart rate (bpm) & $61.0 \pm 9.4$ & $61.5 \pm 9.7$ & \\
\hline Brachial SBP (mmHg) & $112.9 \pm 14.9$ & $112.2 \pm 14.0$ & \\
\hline Brachial DBP (mmHg) & $70.0 \pm 8.3$ & $69.2 \pm 9.2$ & \\
\hline Alx* (\%) & $17.25(-18.5$ to 40$)$ & $11.75(-22$ to 35.5$)$ & 0.004 \\
\hline $\mathrm{PWV}^{*}\left(\mathrm{~m} \mathrm{~s}^{-1}\right)$ & $5.9(4.1-8.4)$ & $5.6(4.1-13.4)$ & \\
\hline
\end{tabular}

Data are mean $\pm S D$. There were no significant differences between the groups unless P-value is stated. *Median (range) cited. HDL, High-density lipoprotein; hsCRP, high-sensitivity C-reactive protein; SBP, systolic blood pressure; DBP, diastolic blood pressure; Alx, augmentation index; PWV, pulse wave velocity.

Table 2

Study 1: results of the multiple regression analysis using augmentation index as the dependent variable $(n=88)$

\begin{tabular}{lrrr}
\hline Predictor & \multicolumn{1}{l}{$\boldsymbol{\beta}$} & $P$-values \\
\hline Age & 0.723 & 0.602 & $<0.001$ \\
Diastolic blood pressure & 0.653 & 0.415 & $<0.001$ \\
Smoking status & 6.106 & 0.223 & $<0.001$ \\
Blood glucose & -1.866 & -0.126 & 0.045 \\
Weight & -0.146 & -0.158 & 0.049 \\
Systolic blood pressure & -0.170 & -0.181 & 0.091 \\
Sex & 4.523 & 0.154 & 0.168 \\
Total : HDL-cholesterol ratio & 0.713 & 0.069 & 0.328 \\
Creatinine & -0.029 & -0.032 & 0.680 \\
Height & -3.882 & -0.028 & 0.787 \\
Alcohol & -0.027 & -0.014 & 0.812 \\
& & & \\
\hline
\end{tabular}

$B$, Unstandardized regression coefficient; $\beta$, standardized regression coefficient. HDL, High-density lipoprotein.

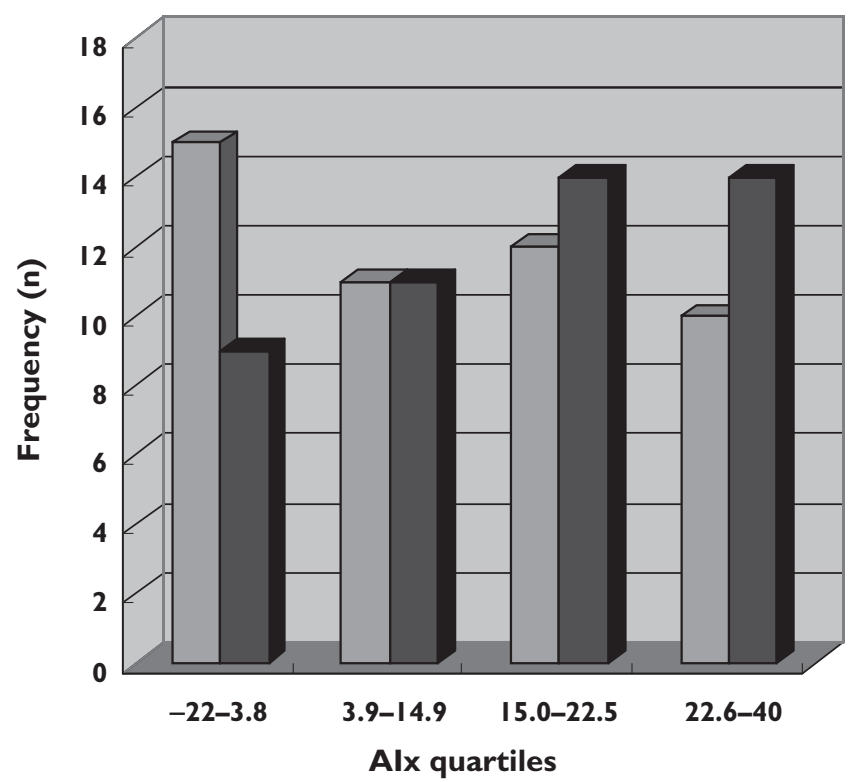

Figure 1

Study 1: augmentation index (Alx) (\%) of age- and sex-matched nonsmokers ( $\square)$ vs. smokers 
Table 3

Study 2: individual patient data recorded at baseline and 4 weeks post quit day $(n=14)$

\begin{tabular}{|c|c|c|c|c|c|c|c|c|}
\hline Age & \multicolumn{4}{|c|}{ Baseline (quit day) } & \multicolumn{4}{|c|}{4 weeks post quit day } \\
\hline \multicolumn{9}{|c|}{ Successful quitters $(\mathrm{n}=10)$} \\
\hline 51.5 & $\mathrm{M}$ & 20 & 12 & 276 & 22 & -92 & -14 & 13 \\
\hline 52.4 & $\mathrm{~F}$ & 23 & 7 & 555 & 29 & -100 & -86 & 19 \\
\hline 65.9 & $\mathrm{~F}$ & 5 & 5 & 99 & 30 & -100 & $+47^{*}$ & 37 \\
\hline 66.6 & $\mathrm{~F}$ & 20 & 1 & 350 & 31 & $+400 \dagger$ & -47 & 27 \\
\hline 67.4 & $\mathrm{~F}$ & 20 & 5 & 130 & 40 & -100 & -34 & 32 \\
\hline 46.8 & $\mathrm{~F}$ & 15 & 6 & 282 & 40 & -83 & -70 & 34 \\
\hline 76.4 & $\mathrm{~F}$ & 5 & 12 & 276 & 26 & -100 & -63 & 23 \\
\hline 54.2 & M & 35 & 11 & 462 & 37 & -91 & -96 & 35 \\
\hline \multicolumn{9}{|c|}{ Relapsed smokers $(\mathrm{n}=4)$} \\
\hline 53.7 & $\mathrm{~F}$ & 20 & 12 & 331 & 35 & -42 & -36 & 35 \\
\hline 59.7 & $\mathrm{~F}$ & 20 & 10 & 361 & 11 & -100 & -42 & 22 \\
\hline 63.8 & M & 35 & 28 & 282 & 40 & -100 & -17 & 48 \\
\hline
\end{tabular}

*Cotinine concentration increased $47 \%$ from baseline due to nicotine replacement therapy; smoking status validated by CO monitor. †Anomalous increase in expired CO to 5 p.p.m. at 4 weeks post quit day (probably due to passive smoking and very low baseline reading).

The change in AIx from baseline to 4 weeks post quit day was significantly different between the quitters and relapsed smokers (Table 3), quitters showing a reduced AIx following 4 weeks of abstinence from smoking (median change 5.0 vs. -9.5 ; difference 14.0, 96\% CI 4.0, 22.0; Mann-Whitney $P=0.013$ ). The change in PWV did not reach significance between the two groups.

\section{Discussion}

Many of the traditional cardiovascular risk factors, including smoking, have been associated with endothelial dysfunction, which may be the pathological process responsible for the development of cardiovascular disease [17]. Basal NO production and other measures of endothelial dysfunction are very difficult to measure non-invasively. However, NO is a potent smooth muscle relaxant and is a principal regulator of arterial smooth muscle tone [18]. AIx and pulse wave velocity are both indices of arterial stiffness that can be measured noninvasively using pulse wave analysis.

Mahmud and Feely have demonstrated that smoking a single cigarette produces an acute increase in arterial stiffness in chronic smokers and nonsmokers [4]. In the same paper, they examined the chronic effects of smoking on AIx in a young population (mean age $23 \pm 5$ years) by comparing 41 smokers vs. 116 nonsmokers. AIx was significantly higher in smokers, indicating increased wave reflection, but PWV and therefore the contribution of aortic arterial stiffness was not measured. In the current study, we used a matched-pair analysis to demonstrate a significantly higher AIx, but not PWV, in smokers. The effects on vascular tone of impaired endothelium-dependent vasodilation, previously demonstrated in smokers, are greater in peripheral muscular arteries than in central elastic arteries, where elastin is the major determinant of arterial stiffness. Thus, the effects of smoking on smooth muscle tone would be expected to affect systemic, but not necessarily aortic arterial stiffness, as our results have shown.

The magnitude of the difference in AIx between smokers and nonsmokers was similar to that observed in the previous study, which used a younger subject population. In addition, our multiple regression model showed that smoking was the strongest predictor of AIx after age and diastolic BP. Our data did not show a doseresponse relationship between difference in smoking and nonsmoking AIx and cigarette consumption, so that light smokers and heavy smokers both had a similar 
elevation in AIx compared with nonsmoking counterparts. This may suggest that smoking 10 cigarettes per day (the cut-off for inclusion as a smoker) is enough to induce marked endothelial dysfunction. Indeed, recent work by Barua et al. [2] found that basal NO production and eNOS expression and activity were all impaired to the same extent in light and heavy smokers.

A number of other factors are known to influence AIx and although efforts were made to match the smoking and control groups as closely as possible, HDLcholesterol, total:HDL ratio and alcohol consumption were still significantly higher among the smoking cohort. The relationship between smoking and these variables has been reported previously [16, 19]. However, in our multiple regression model, total:HDL ratio and alcohol intake showed no significant association with either AIx or PWV, whereas smoking status was still a significant independent determinant of AIx $(P<0.001$, Table 2$)$.

In study 1 , increased systemic arterial stiffness was demonstrated in healthy smokers when compared with nonsmoking controls. The next logical question is whether this effect is reversible on quitting smoking. There is some evidence that an improvement in endothelial function follows smoking cessation. One study demonstrated significantly reduced plasma NO levels in current smokers but no difference in former smokers and never smokers [20]. The time since stopping smoking among the former smokers ranged from 6 months to 12 years and there was no association with basal plasma NO levels. This suggests that most of the improvement in endothelial function may occur in the first few months after quitting [20]. This corroborated previously published data demonstrating an improved flow-mediated dilation in former smokers compared with current smokers, which approached the levels of nonsmoking controls [21]. In keeping with the observations of Node et al. [20], flow-mediated dilation was not related to time since smoking cessation among the former smokers.

Contrary to previously reported findings, our data failed to show an association between hsCRP levels and smoking status $[22,23]$. However, both studies involved more than 700 subjects whilst our data involved only a subset of the study 1 participants $(n=52)$. The significant finding of hsCRP levels predicting aortic PWV in healthy volunteers is consistent with a recent study which also employed the SphygmoCor system to perform PWA [24].

In study 2, we found that AIx decreased after 4 weeks of smoking cessation in successful quitters. Conversely, subjects who resumed regular smoking demonstrated an overall increase in AIx at 4 weeks and the difference in AIx response after 4 weeks was significantly different between the quitters and smokers $(P=0.013)$. An inter- esting finding was that AIx increased, rather than remaining unchanged, at 4 weeks with continued smoking. This may be explained by an acute effect of smoking on AIx [4, 5], since all subjects who were recruited into study 2 were asked to refrain from smoking overnight before attending the clinical unit, but could smoke freely up to their study visit if they had relapsed at 4 weeks. These measurements could therefore reflect an acute effect of smoking on systemic arterial stiffness.

Our findings suggest an almost immediate recovery of endothelial function, although this is difficult to determine in such small numbers and without further follow-up. A study of similar design, but not measuring PWA, indicated an improvement in basal NO production after only 1 week of smoking cessation [25]. It monitored the change in exhaled NO levels among 25 smoking cessation clinic attendees over an 8-week timescale. At baseline smokers showed a reduced basal exhaled NO compared with matched nonsmoking controls. Ten of the 25 smokers were abstinent after 8 weeks and showed an increase towards normal exhaled NO levels at 1 week, followed by levels at 8 weeks that were not significantly different from those of the nonsmokers. It should be noted that the exhaled NO was probably the product of NOS in lung epithelial cells rather than the vascular endothelium; however, recovery of epithelial NOS function may indicate similar improvement in the endothelium. Further largescale studies are required to elucidate the time-related effects of smoking cessation on arterial properties, including follow-up beyond 12 weeks. In addition, attempts should be made to quantify the improvement by comparison with that of a healthy nonsmoker.

In both the above studies, arterial stiffness measured by pulse wave analysis was used to demonstrate impaired basal arterial tone among smokers, an indicator of endothelial dysfunction. It may provide a predictive role in risk assessment, a surrogate endpoint in clinical trials and a target for therapeutic intervention [9]. However, one limitation of the SphygmoCor apparatus was that readings could not be obtained for 14 subjects, due to a combination of faint radial, carotid or femoral pulses, and rejection of the pulse wave trace by the software's quality control algorithms. Such measurement difficulties have been cited previously in the literature [15]. In addition, there is some debate among experts in the field regarding the use of a single general transfer function to estimate central pulse waveforms [26, 27].

In summary, these studies have shown that chronic smoking affects systemic arterial stiffness, as measured by AIx, in healthy subjects free from other cardiovascular risk factors. Chronic smoking does not seem to affect carotid-femoral PWV, which may indicate that the major- 
ity of the effect on arterial stiffness is mediated through the effect of smoking on endothelial dysfunction. The effect of chronic smoking on AIx may be partly reversed after only 4 weeks of smoking cessation. Additional investigations are required to confirm the long-term effects of smoking cessation on PWA and endothelial function. If improvement in arterial function can be demonstrated, then use of AIx in the clinical setting may prove a useful motivational tool in smoking cessation.

\section{Conflict of interest}

\section{None to declare.}

\section{References}

1 Holbrook JH, Grundy SM, Hennekens CH, Kannel WB, Strong JP. Cigarette smoking and cardiovascular diseases. A statement for health professionals by a task force appointed by the steering committee of the American Heart Association. Circulation 1984; 70: 1114A-1117A.

2 Barua RS, Ambrose JA, Eales-Reynolds LJ, DeVoe MC, Zervas JG, Saha DC. Heavy and light cigarette smokers have similar dysfunction of endothelial vasoregulatory activity. J Am Coll Cardiol 2002; 39: 1758-63.

3 Gottlieb SO. Cardiovascular benefits of smoking cessation. Heart Dis Stroke 1992; 1: 173-5.

4 Mahmud A, Feely J. Effect of smoking on arterial stiffness and pulse pressure amplification. Hypertension 2003; 41: 183-7.

5 Vlachopoulos C, Kosmopoulou F, Panagiotakos D, loakeimidis N, Alexopoulos N, Pitsavos C, Stefanadis C. Smoking and caffeine have a synergistic detrimental effect on aortic stiffness and wave reflections. J Am Coll Cardiol 2004; 44: 1911-7.

6 Mack WJ, Islam T, Lee Z, Selzer RH, Hodis HN. Environmental tobacco smoke and carotid arterial stiffness. Prev Med 2003; 37: 148-54.

7 Mahmud A, Feely J. Effects of passive smoking on blood pressure and aortic pressure waveform in healthy young adults-influence of gender. Br J Clin Pharmacol 2004; 57: 37-43.

8 Rosenberg L, Kaufman D, Helmrich S, Shapiro S. The risk of myocardial infarction after quitting smoking in men under 55 years of age. New Engl J Med 1985; 313: 1511-4.

9 Luscher TF, Noll G. Endothelial function as an end point in international trials: concepts, methods and current data. J Hypertens 1996; 145: S111-S121.

10 O'Rourke MF, Pauca A, Jing Jang X. Pulse wave analysis. Br J Clin Pharmacol 2001; 51: 507-22.

11 Boutouyrie P, Tropeano Al, Asmar R, Gautier I, Benetos A, Lacolley $P$, Laurent $S$. Aortic stiffness is an independent predictor of primary coronary events in hypertensive patients. A longitudinal study. Hypertension 2002; 39: 10-5.

12 Wilkinson IB, Hall IR, MacCallum H, Mackenzie IS, McEniery CM, Van der Arend BJ, Shu YE, MacKay LS, Webb DJ, Cockcroft JR. Pulse-wave analysis: clinical evaluation of a noninvasive, widely applicable method for assessing endothelial function. Arterioscler Thromb Vasc Biol 2002; 22: 147-52.
13 Smith JC, Evans LM, Wilkinson I, Goodfellow J, Cockcroft JR, Scanlon MF, Davies JS. Effects of $\mathrm{GH}$ replacement on endothelial function and large-artery stiffness in GH-deficient adults: a randomized, double-blind, placebo-controlled study. Clin Endocrinol 2002; 56: 493-501.

14 Stolker AM, Niesing W, Hogendoorn EA, Rambali AB, Vleeming W. Determination of nicotine and cotinine in rat plasma by liquid chromatography-tandem mass spectrometry. J Chromatogr A 2003; 1020: 35-43.

15 Wilkinson IB, Fuchs SA, Jansen IM, Spratt JC, Murray GD, Cockcroft JR, Webb DJ. Reproducibility of pulse wave velocity and augmentation index measured by pulse wave analysis. J Hypertens 1998; 16: 2079-84.

16 Smulyan H, Marchais SJ, Pannier B, Guerin AP, Safar ME, London $\mathrm{GM}$. Influence of body height on pulsatile arterial hemodynamic data. J Am Coll Cardiol 1998; 31: 1103-9.

17 Ross R. Atherosclerosis-an inflammatory disease. N Engl J Med 1999; 340: 115-26.

18 De Meyer GYR, Herman AG. Vascular endothelial dysfunction. Prog Cardiovasc Dis 1997; 34: 324-42.

19 Price JF, Mowbray PI, Lee AJ, Rumley A, Lowe GD, Fowkes FG. Relationship between smoking and cardiovascular risk factors in the development of peripheral arterial disease and coronary artery disease: Edinburgh Artery Study. Eur Heart J 1999; 20: 344-53.

20 Node K, Kitakaze M, Yoshikawa H, Kosaka H, Hori M. Reversible reduction in plasma concentration of nitric oxide induced by cigarette smoking in young adults. Am J Cardiol 1997; 79: 1538-41.

21 Celermajer DS, Sorensen KE, Georgakopoulos D, Bull C, Thomas O, Robinson J, Deanfield JE. Cigarette smoking is associated with dose-related and potentially reversible impairment of endothelium-dependent dilation in healthy young adults. Circulation 1993; 88: 2149-55.

22 Danesh J, Muir J, Wong Y-K, Ward M, Gallimore JR, Pepys MB. Risk factors for coronary heart disease and acute-phase proteins. Eur Heart J 1999; 20: 954-9.

23 Saito $M$, Ishimitsu $T$, Minami J, Ono $H$, Ohrui M, Matsuoka $H$. Relations of plasma high-sensitivity C-reactive protein to traditional cardiovascular risk factors. Atherosclerosis 2003; 167: 73-9.

24 Yasmin McEniery CM, Wallace S, Mackenzie IS, Cockcroft JR, Wilkinson IB. C-reactive protein is associated with arterial stiffness in apparently healthy individuals. Arterioscler Thromb Vasc Biol 2004; 24: 969-74.

25 Robbins RA, Millatmal T, Lassi K, Rennard S, Daughton D. Smoking cessation is associated with an increase in exhaled nitric oxide. Chest 1997; 112: 313-8.

26 Hope SA, Tay DB, Meredith IT, Cameron LD. Use of arterial transfer functions for the derivation of central aortic waveform characteristics in subjects with type 2 diabetes and cardiovascular disease. Diabetes Care 2004; 27: 746-51.

27 Millasseau SC, Patel SJ, Redwood SR, Ritter JM, Chowienczyk PJ. Pressure wave reflection assessed from the peripheral pulse. Is a transfer function necessary? Hypertension 2003; 41: 101620. 